Supporting Information

\title{
Visualizing Chemo-Mechanical Degradation of a Solid-State Battery Electrolyte
}

Jared Tippens ${ }^{1}$, John C. Miers ${ }^{1}$, Arman Afshar ${ }^{3}$, John A. Lewis ${ }^{2}$, Francisco Javier Quintero Cortes $^{2}$, Haipeng Qiao ${ }^{1}$, Thomas S. Marchese ${ }^{2}$, Claudio V. Di Leo ${ }^{3}$, Christopher Saldana ${ }^{1}$, Matthew T. McDowell ${ }^{1,2^{*}}$

${ }^{1}$ George W. Woodruff School of Mechanical Engineering, Georgia Institute of Technology, 801 Ferst Drive, Atlanta, GA, 30332

${ }^{2}$ School of Materials Science and Engineering, Georgia Institute of Technology, 771 Ferst Drive, Atlanta, GA, 30332

${ }^{3}$ The Daniel Guggenheim School of Aerospace Engineering, Georgia Institute of Technology, 270 Ferst Drive, Atlanta, GA, 30332

*Corresponding Author: mattmcdowell@gatech.edu 


\section{EXPERIMENTAL METHODS}

Synthesis: LAGP was synthesized with a sol-gel process. $0.2 \mathrm{M}$ citric acid and $0.8 \mathrm{M}$ $\mathrm{Ge}\left(\mathrm{OC}_{2} \mathrm{H}_{5}\right)_{4}$ were mixed in water on a hot plate for $20 \mathrm{~h}$ at a temperature of $80{ }^{\circ} \mathrm{C}$. Stoichiometric amounts of $\mathrm{LiNO}_{3}, \mathrm{Al}\left(\mathrm{NO}_{3}\right)_{3} \cdot 9 \mathrm{H}_{2} \mathrm{O}$, and $\mathrm{NH}_{4} \mathrm{H}_{2} \mathrm{PO}_{4}$ were added to the solution and stirred for 30 min. Ethylene glycol was then added in the same molar ratio as the citric acid, and the temperature was ramped to $120{ }^{\circ} \mathrm{C}$ and held for an additional $30 \mathrm{~min}$. The solution was gradually dried by ramping the temperature to $150{ }^{\circ} \mathrm{C}$ for $30 \mathrm{~min}$ and $170{ }^{\circ} \mathrm{C}$ for $60 \mathrm{~min}$. This resulted in a dry powder that was then heated in a furnace at $500{ }^{\circ} \mathrm{C}$ for $4 \mathrm{~h}$ and then at $800{ }^{\circ} \mathrm{C}$ for $5 \mathrm{~h}$. This powder was ground using a mortar and pestle and then pressed into pellets approximately $9.5 \mathrm{~mm}$ in diameter and $1 \mathrm{~mm}$ thick. These pellets were sintered in a furnace in air for 11 hours at $900{ }^{\circ} \mathrm{C}$, resulting in a diameter decrease to $\sim 8.6 \mathrm{~mm}$.

Cell Fabrication: In an Ar-filled glove box, $0.75 \mathrm{~mm}$ thick lithium metal foil was punched into $1 / 4$-in diameter disks and pressed onto both sides of an LAGP pellet. This stack was then loaded into a 2032 stainless steel coin cell with a steel spacer and conductive foam on one side. The coin cell was then crimped to a pressure of $500 \mathrm{psi}$. A slightly modified fabrication procedure was used for the experiments shown in Figs. $3 a$ and $3 c$, d. Here, the conductive foam was not used, and three individual lithium foil disks were instead used on both sides of the LAGP. The thicker lithium allowed the top and bottom surfaces of the SSE to be clearly resolved during CT imaging. Coin cell constructions are shown in Fig. S23.

Electrochemistry: A Bio-Logic SP200 potentiostat was used to apply either a constant current or constant voltage during the 1-h cycles. The currents or voltages applied for the data presented herein are denoted in Fig. 2a and Fig. S3. The electrochemical test was paused after each differently-colored region in these figures to perform a CT scan. EIS was conducted before and 
after each CT scan with a frequency sweep from $3 \mathrm{MHz}$ to $2 \mathrm{~Hz}$ and a sine wave amplitude of 5 $\mathrm{mV}$. All fabricated cells were allowed to sit for $24 \mathrm{~h}$ under open circuit conditions before electrochemical testing to allow the epoxy used for mounting to fully cure.

X-ray Imaging: Imaging was carried out using a Zeiss Metrotom $800 \mathrm{CT}$ instrument. A voltage and current of $130 \mathrm{kV}$ and $49 \mu \mathrm{A}$ were used. Each projection image was acquired over 500 ms and then averaged with another image from the same angular position for a total of $1000 \mathrm{~ms}$ per projection. For the experiment detailed in Fig. 2a, each tomographic scan consisted of 1760 total 2D images collected over a rotational sweep of $360^{\circ}$. For the experiment detailed in Fig. 3c and d, the scan was optimized to collect 660 total images over the $360^{\circ}$ rotation to ensure at least full Nyquist sampling [1].

Image Reconstruction and Slicing: The tomographic data were reconstructed using a filtered back projection algorithm with a Shepp-Logan filter. The raw 3D volumes generated by each scan were analyzed in VGSTUDIO MAX 3.1, a visualization and analysis software for CT data created by Volume Graphics Inc. Proper segmentation of the pellet surface geometry required high local contrast; as such, the region surrounding the LAGP pellet was isolated from the primary volume with the specification of a region of interest around the pellet volume. To ensure that the same region was used for each scan of the SSB, a template was created large enough to accommodate the lithium and the LAGP pellet. The isolated volume containing the LAGP pellet and lithium was exported into image stacks in the $\mathrm{XY}, \mathrm{YZ}$, and $\mathrm{XZ}$ planes with each image representing one voxel of thickness in the direction normal to each respective image plane.

Data Analysis: The data collected from the in-situ experiments were used to construct 3D crack networks as seen in Fig. 2c. The MATLAB "imadjust" function was used to optimize contrast of the 2D slices to differentiate between the cracked and uncracked regions. Then, ImageJ 
software was used to segment each pixel in the images into categories of uncracked, cracked, or background via the trainable weka segmentation tool. This was achieved by training a "class" in the software, a process that involves manually labeling different regions. After the classes were applied to segment the images, the images were filtered of noise to produce the binary crack images within the LAGP volume seen in Fig. 2c.

The damaged area in Fig. 3c was quantified by measuring the width of cracked regions that traverse $\mathrm{Li}^{+}$ion flow pathways (i.e., along the axis of the cylindrical pellet) and dividing by the total contact area between LAGP and lithium. A sample of 11 cross-sectional image slices from each tomogram were segmented into areas that were cracked or uncracked. This 11-slice representative sample was employed to estimate the damaged area throughout the entire pellet.

Finite Element Analysis Simulations: See the end of the SI document for full details on finite element analysis. 


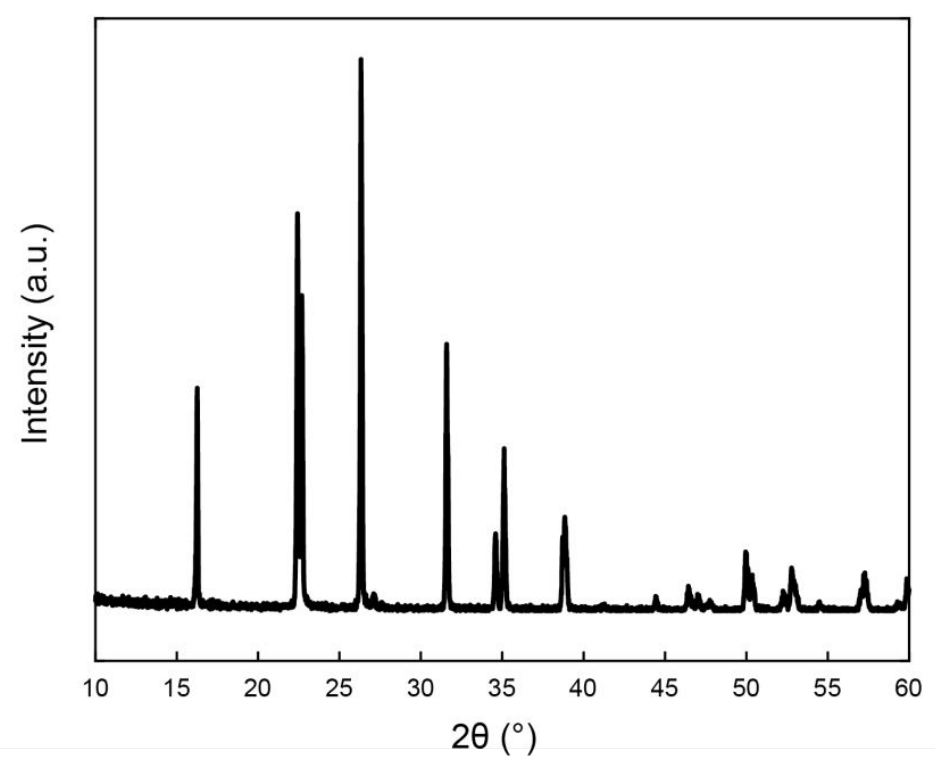

Figure S1: XRD of pristine LAGP showing intensity peaks characteristic of the rhombohedral crystal structure.

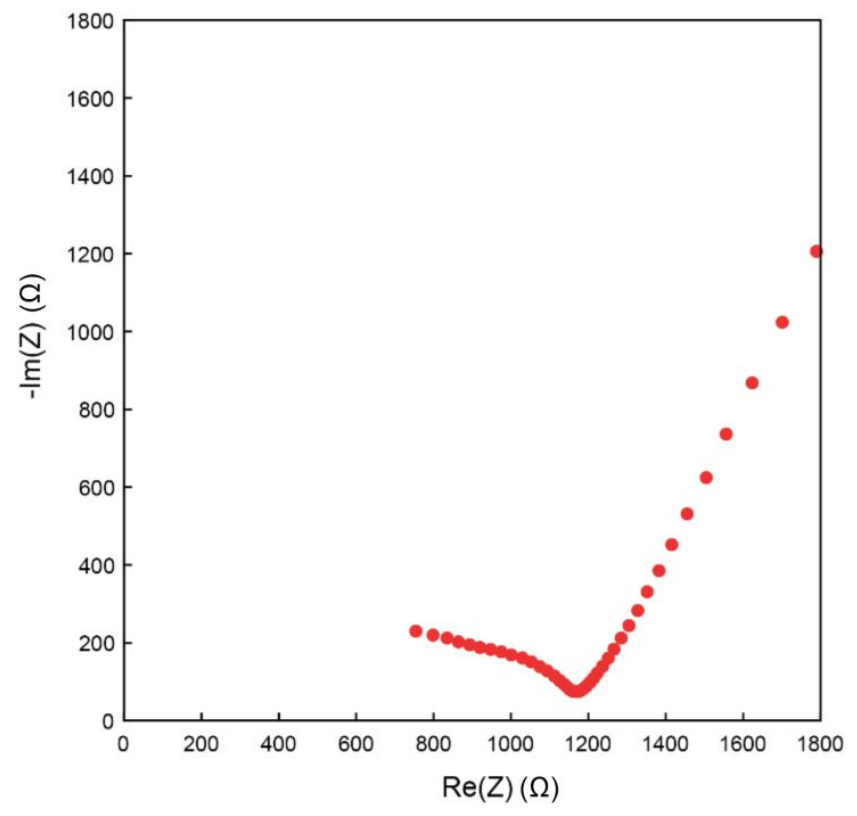

Figure S2: Nyquist plot from a symmetric Au/LAGP/Au cell. 


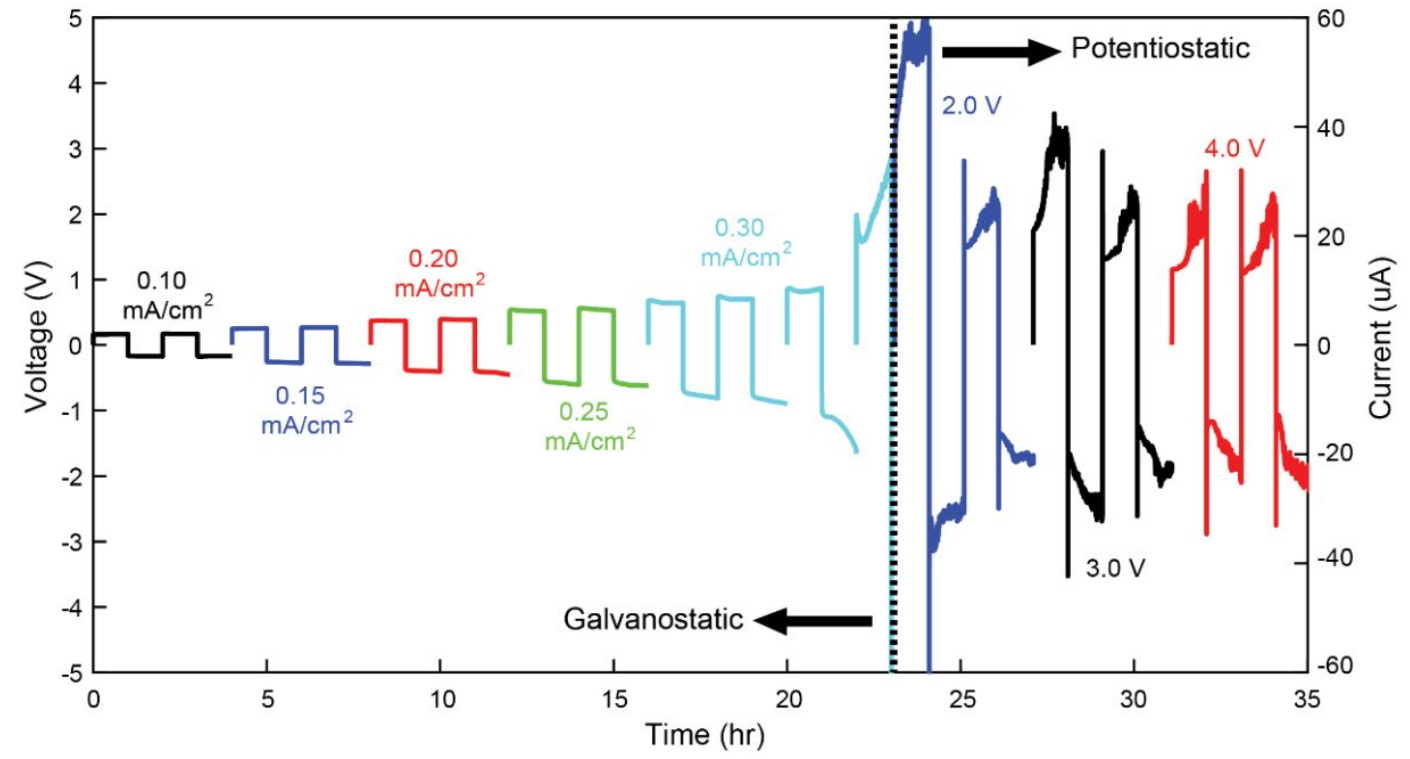

Figure S3: Electrochemical data from the experiment shown in Fig. 1 and Fig. 3c-d. 
Table S1: Table summarizing the electrochemical and mechanical degradation of the LAGP pellet shown in Fig. 2 in the main text over $52 \mathrm{~h}$ of electrochemical cycling. Column 1 shows the total amount of cycling time, corresponding to Fig. 2a. Column 2 shows the 2D image slices for all scans without the initial $\sim 24 \mathrm{~h}$ of scans that do not show changes in morphology. Column 3 shows the 3D-rendered crack networks. Column 4 shows the change in crack volume between scans. Column 5 shows the charge transferred between scans. Column 6 shows the change in impedance between scans, as measured with EIS. Column 7 shows the number of new cracks detected in each scan. The slight decrease in impedance after initial cycling may be due to improved contact due to initial interphase formation.

\begin{tabular}{|c|c|c|c|c|c|c|}
\hline $\begin{array}{c}\text { Total } \\
\text { Cycle } \\
\text { Time (h) }\end{array}$ & Mid Slice & 3D Cracks & $\begin{array}{c}\Delta \text { Crack } \\
\text { Volume } \\
\left(\mathrm{mm}^{3}\right)\end{array}$ & $\begin{array}{c}\text { Charge } \\
\text { Transferred } \\
\text { (mAh) }\end{array}$ & $\begin{array}{c}\Delta \\
\text { Impedance } \\
(\Omega) \\
\end{array}$ & $\begin{array}{c}\Delta \\
\text { Number } \\
\text { of Cracks }\end{array}$ \\
\hline 0 & & & 0 & 0 & 0 & 0 \\
\hline 24 & & & 0.089 & 2.179 & $-1,200$ & 2 \\
\hline 28 & & & 0.24 & 0.5447 & 1,500 & 2 \\
\hline 32 & & & 1.667 & 0.5447 & 22,700 & 42 \\
\hline 36 & & & 0.6158 & 0.1540 & 31,000 & 11 \\
\hline 40 & & & 0.8545 & 0.1241 & 35,000 & 7 \\
\hline 44 & & & 0.7812 & 0.1205 & 75,000 & 8 \\
\hline 48 & & & 1.773 & 0.1131 & 150,000 & 10 \\
\hline 52 & & & 1.214 & 0.6640 & 140,000 & 5 \\
\hline
\end{tabular}




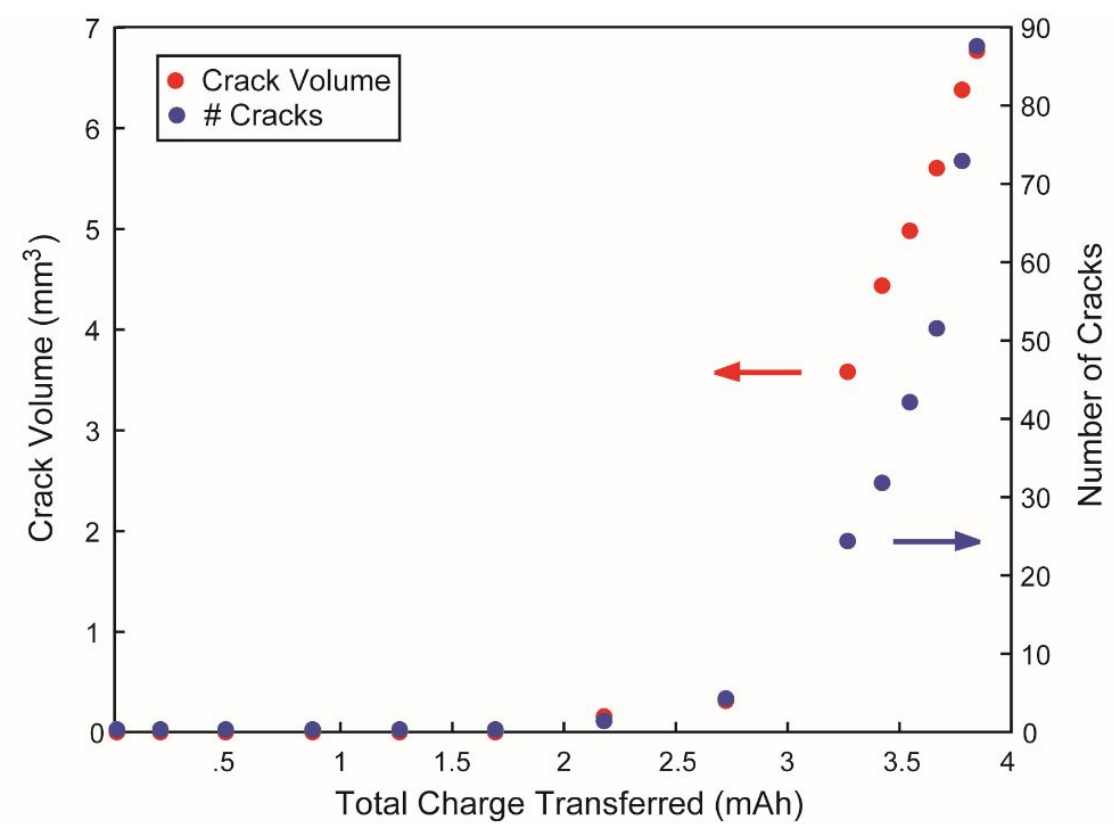

Figure S4: Graph of the number of cracks and total crack volume as a function of charge transferred for the data in Fig. 2 of the main text. There is a significant jump in both crack volume and number of cracks after $\sim 3.3 \mathrm{mAh}$ of charge is passed.
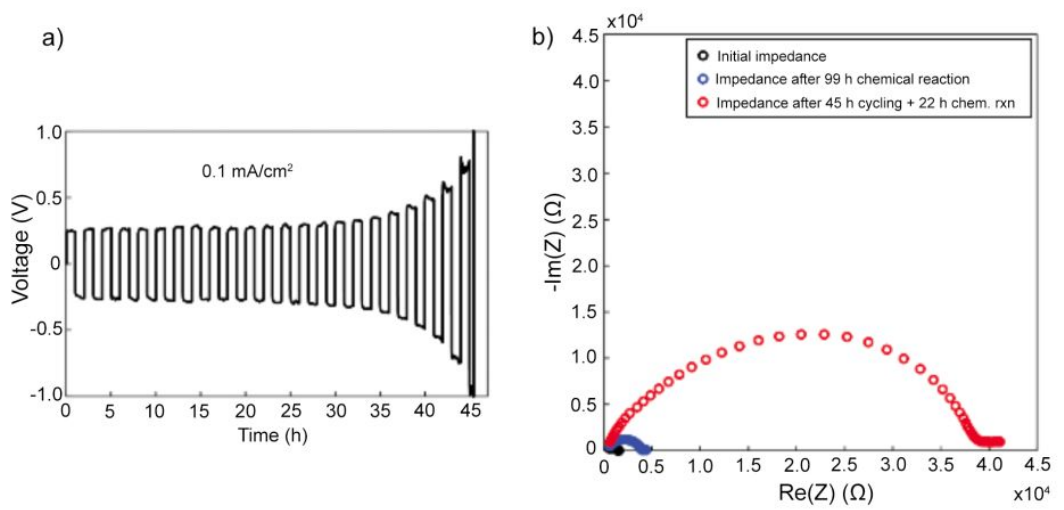

Figure S5: Electrochemistry from an ex-situ experiment to examine interphase growth, with images shown in Fig. 3a. This cell was constructed and held at open circuit for $99 \mathrm{~h}$, then cycled galvanostatically for $45 \mathrm{~h}$, and then held at open circuit for an additional $22 \mathrm{~h}$. (a) Galvanostatic data at $0.10 \mathrm{~mA} \mathrm{~cm}^{-2}$. (b) EIS after cell construction (black), after holding at open circuit (blue), and after the full experiment (red). 


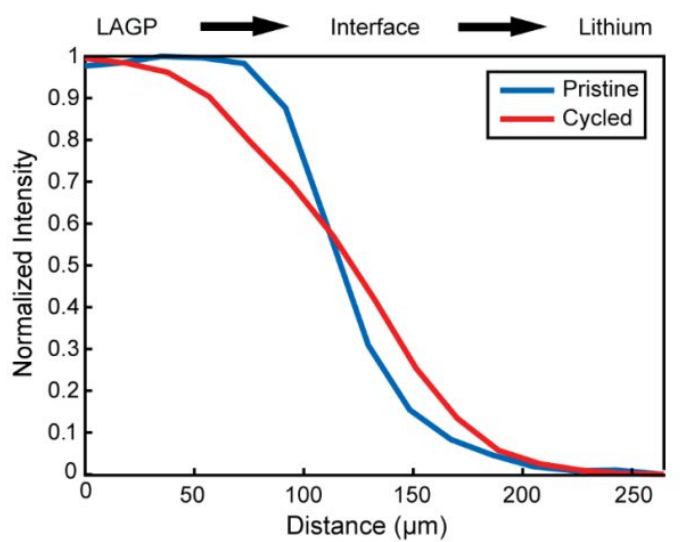

Figure S6: Averaged image intensity across the Li/LAGP interface over a wide area of the pellet in Fig. 3a after reaction shows that the reacted interphase results in a shallower gradient; this indicates that the interphase is detected across much of the pellet.

(a)

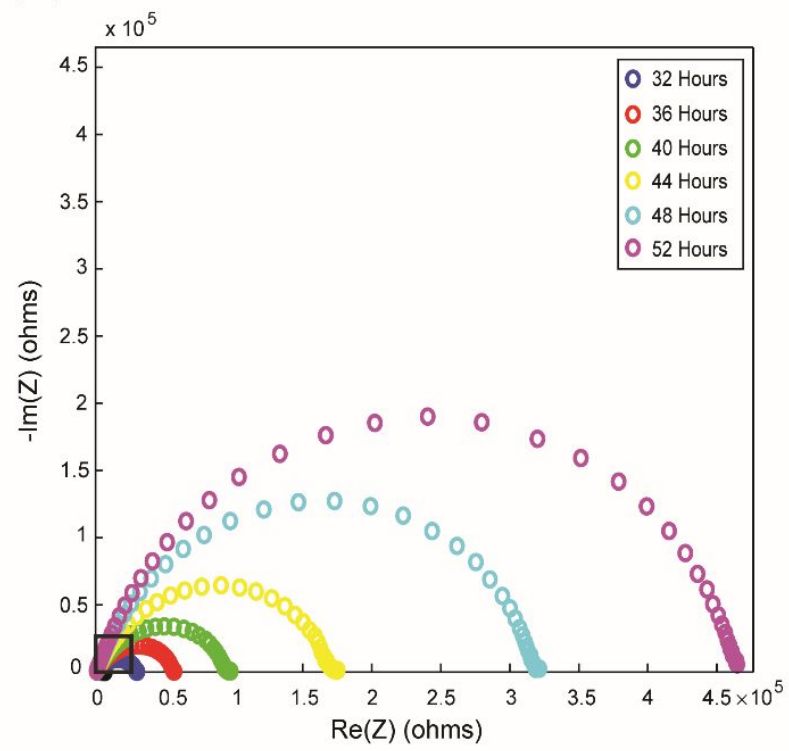

(b)

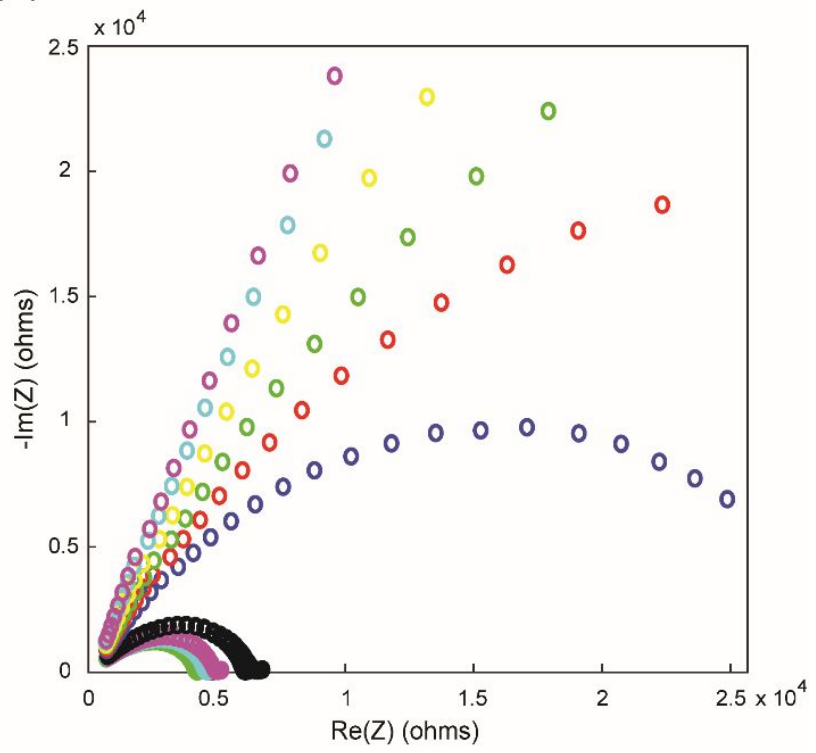

Figure S7: EIS data from the electrochemical test presented in Fig. 2a. (a) A plot showing all of the EIS data for the entire experiment. (b) A magnified portion of the EIS plot in (a) showing the lowerimpedance region. 
(a)

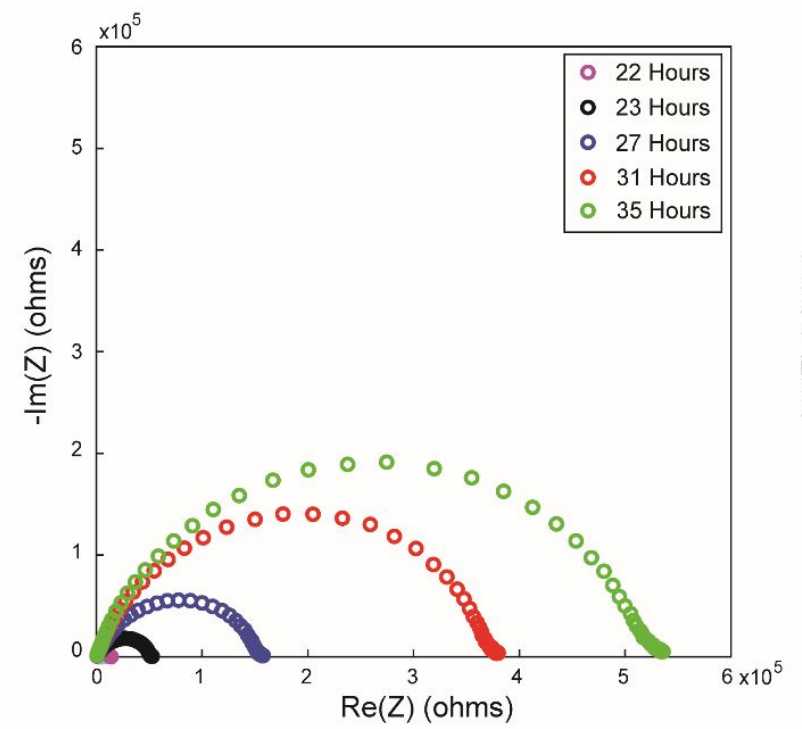

(b)

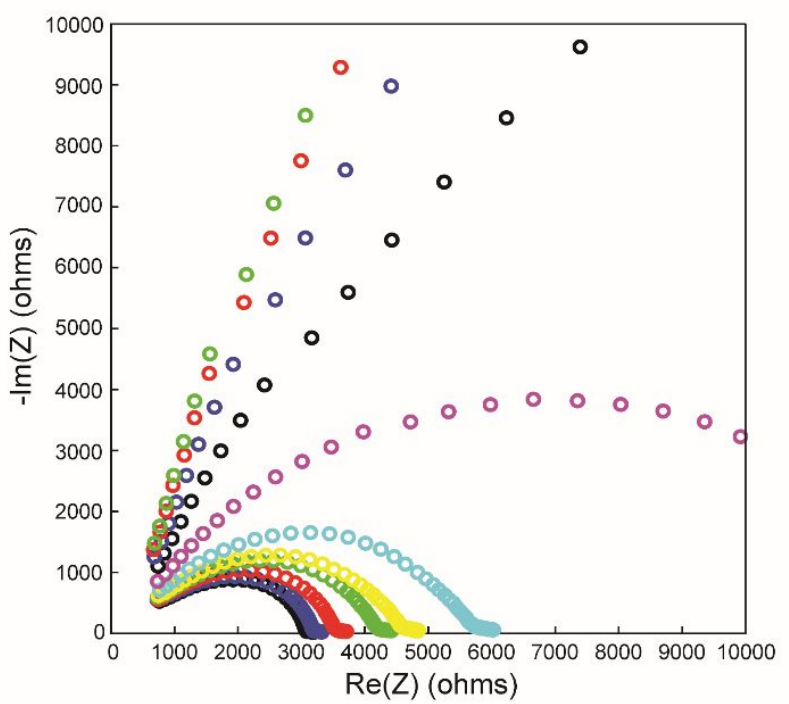

Figure S8: EIS data from the experiment detailed in Fig. 3c-d. (a) A plot showing all of the EIS data for the entire experiment. (b) A magnified portion of the EIS plot in (a) showing the lower-impedance region.

(a)

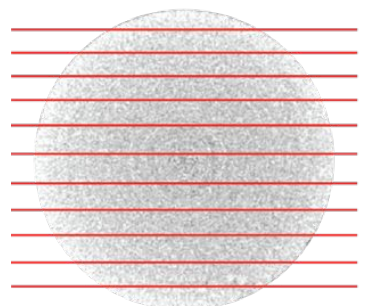

(b)

70\% Damaged, 30\% Undamaged

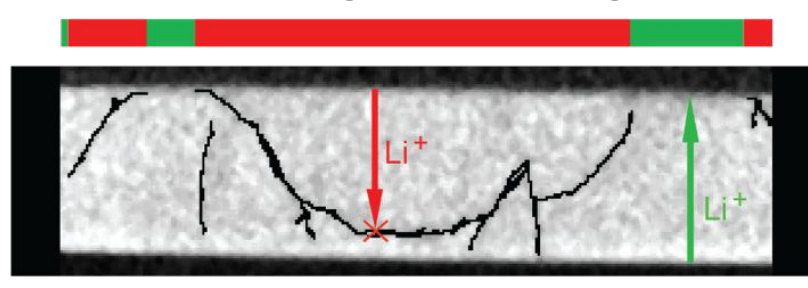

Figure S9: Damaged area calculation procedure for the graph in Fig. 3c. (a) Locations of the 11 crosssectional slices from which the damaged area was calculated for each tomogram. (b) Diagram showing how the damaged area was computed for each slice. 
(a)

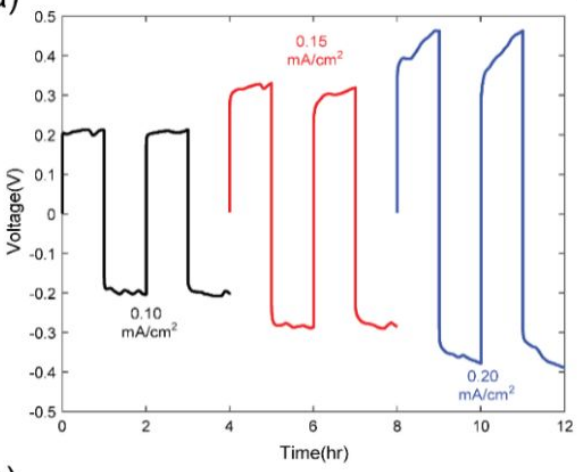

(b)

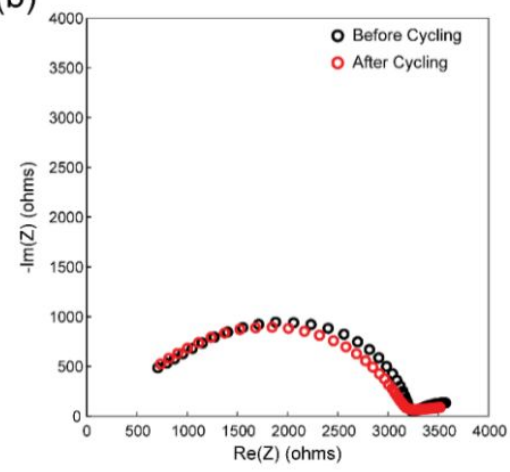

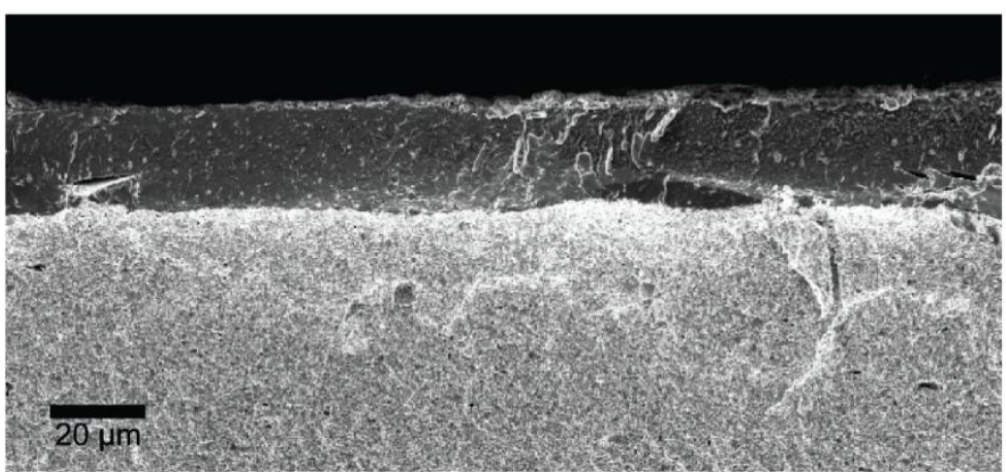

Figure S10: Data from a symmetric cell galvanostatically cycled ex situ. The cell was held at open circuit for $\sim 24 \mathrm{~h}$ and then electrochemically tested. (a) Galvanostatic test at different currents after the $\sim 24$ h open-circuit hold. (b) EIS showing no significant increase in total cell impedance just after cell construction and at the end of cycling. (c) SEM image showing the growth of a 20-25 $\mu \mathrm{m}$ thick, mostly uniform interphase after this process. 
(a)

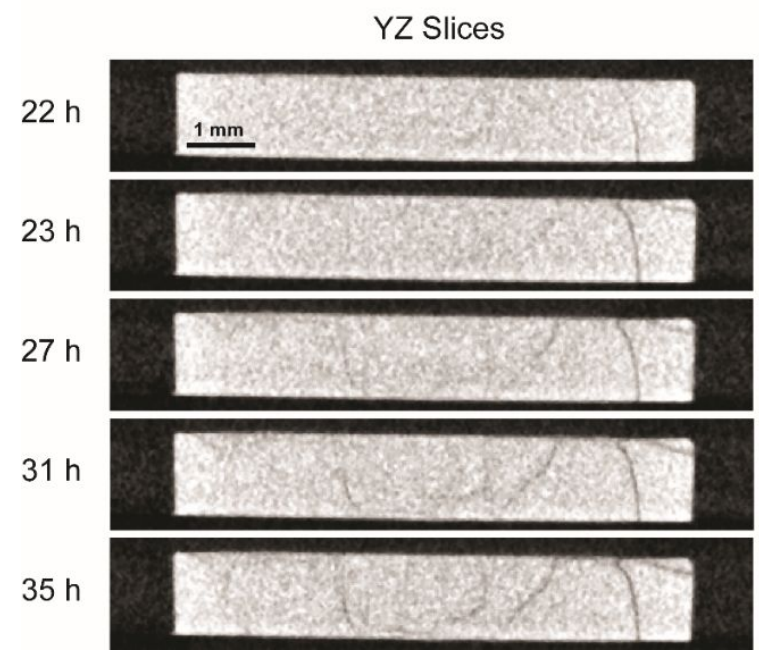

(b)

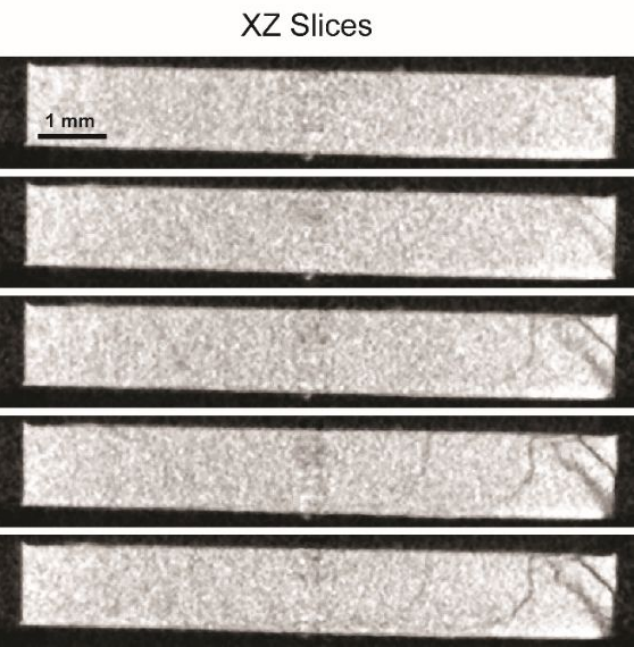

Figure S11: Image slices showing fracture initiation and progression with cycling from the same locations in the pellet as shown in Fig. 3d. (a) Slices in the YZ plane. (b) Slices in the XZ plane.

(a)

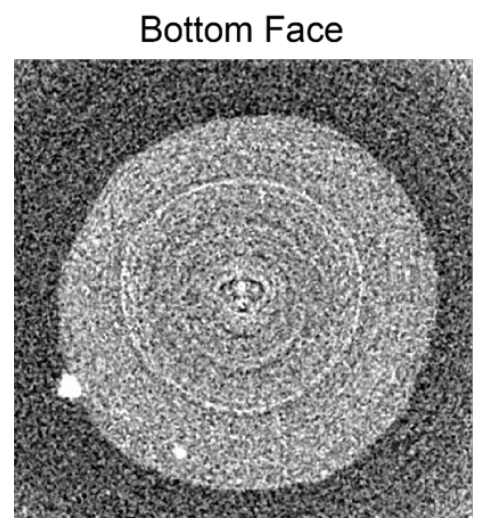

(b)

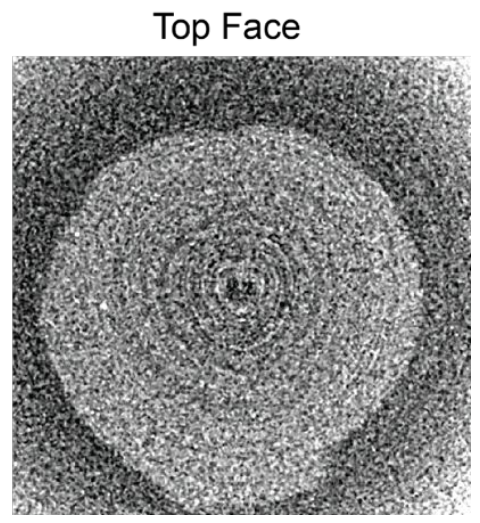

Figure S12: Images showing 2D slices of the lithium electrode from the experiments shown in Fig. 3c-d. (a) Shows the lithium electrode on the bottom surface of the LAGP. (b) Shows the lithium electrode on the top surface of the LAGP. The lithium area is between $75 \%$ and $80 \%$ of the LAGP area (not shown). 


\section{Finite Element Modeling}

The finite element analysis simulations were carried out by modeling half of an axisymmetric disk. The simulation domain is shown schematically in Fig. S13. Nodes along the left edge, which is on the axis of symmetry, are prescribed zero horizontal (radial) displacement, while nodes along the bottom edge are prescribed zero vertical displacement to capture the symmetry boundary condition. All other surfaces are traction free. We prescribe a constant flux over $80 \%$ of the top surface of the body mimicking the contact of the LAGP with lithium.

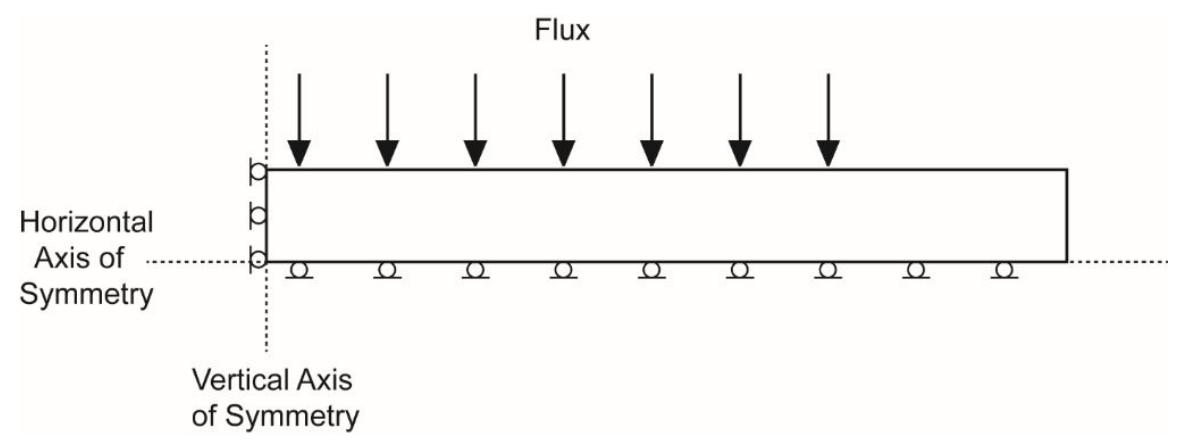

Figure S13: Geometry and boundary condition for the finite element simulations.

The actual finite element mesh is shown in Fig. S14. The top layer near the flux boundary condition is structured with a high density of elements to accurately resolve the gradients in concentration and stress which develop around those zones. The mesh has approximately 100,000 elements.

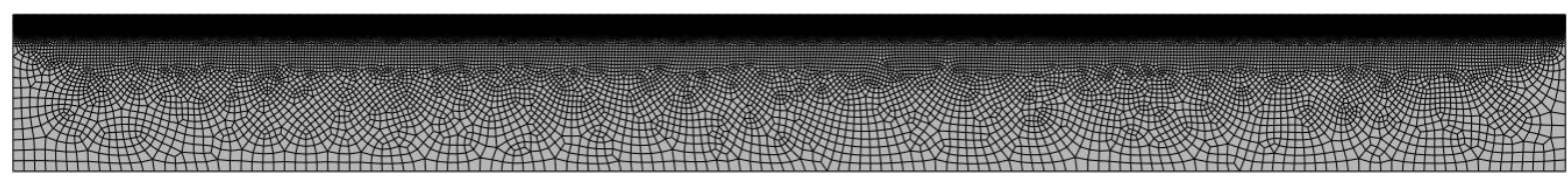

Figure S14: Finite element mesh, refined near applied flux edge to improve accuracy.

For completeness, we briefly summarize here the coupled deformation-diffusion theory used in the simulations. The theory closely follows our previous work [2] with changes mostly to the constitutive choices in the theory. To capture the coupled deformation-reaction process, the deformation gradient $\mathbf{F}$ is decomposed into elastic $\mathbf{F}^{\mathrm{e}}$, plastic $\mathbf{F}^{\mathrm{p}}$, and chemical $\mathbf{F}^{\mathrm{c}}$ distortions through

$$
\mathbf{F}=\mathbf{F}^{\mathrm{e}} \mathbf{F}^{\mathrm{p}} \mathbf{F}^{\mathrm{c}}
$$


In such a decomposition, the quantities $\mathbf{F}^{\mathrm{p}}$ and $\mathbf{F}^{\mathrm{c}}$ jointly capture the kinematics associated with the expansion due to the chemical reaction, captured through $\mathbf{F}^{\mathrm{c}}$, and the concurrent inelastic flow (i.e. bond breaking and forming) captured through $\mathbf{F}^{\mathrm{p}}$. Any resulting elastic deformation, either in the reacted phase or the pristine phase, is captured through the distortion $\mathbf{F}^{\mathrm{e}}$. In accordance with the description above, the chemical distortion is coupled to the concentration of species through

$$
\mathbf{F}^{\mathrm{c}}=\left(J^{\mathrm{c}}\right)^{1 / 3} \mathbf{1}, \quad J^{\mathrm{c}}=1+\Omega c_{R}
$$

Where $\Omega$ is the partial molar volume of $\mathrm{Li}$ in the reacted phase and $c_{R}$ is the molar concentration per unit reference volume. This term couples the expansion due to the chemical reaction with deformation. The Cauchy stress measure $\mathbf{T}$ and the Mandel stress measure $\mathbf{M}^{\mathrm{e}}$ are the same as those used by Di Leo et al. in reference [2]. The concentration-dependent elastic modulus (Young's modulus) vary from a pristine to a reacted value through

$$
E=a E_{\text {reacted }}+(1-a) E_{\text {pristine }}, a=\frac{\Omega \cdot c_{\mathrm{R}, \max } \cdot \bar{c}}{\Omega \cdot c_{\mathrm{R}, \max } \cdot \bar{c}+1}
$$

Here, 'a' denotes the atomic fraction of Li atoms and $\bar{c}=c_{\mathrm{R}} / c_{\mathrm{R}, \max }$, where $c_{\mathrm{R} \text {,max }}$ is the molar concentration in the maximally lithiated LAGP phase. $E_{\text {reacted }}$ is the Young's modulus of the reacted interphase, and $E_{\text {pristine }}$ is the Young's modulus of the LAGP material. This concentration-dependent behavior is based on the experimental observations of Sethuraman et al. [3] for experiments on the lithiation of a-Si, a wellstudied anode material for Li-ion batteries, which is rather similar to the interphase growth process modeled here. It is unclear exactly how material properties vary in the reacted LAGP phase, and as such, this specific constitutive choice should be refined as further experimental evidence for the properties of LAGP become available. A sensitivity analysis for the elastic moduli values is presented subsequently. The elastic and chemical expansion behavior is characterized by the following material properties:

$$
\begin{gathered}
E_{\text {pristine }}=115 \mathrm{GPa}, E_{\text {reacted }}=11.0 \mathrm{GPa}, v=0.3, \\
\Omega C_{\mathrm{R}, \max }=1.30, c_{\mathrm{R}, \max }=0.0484 \times 10^{6} \mathrm{~mol} / \mathrm{m}^{3} .
\end{gathered}
$$

Here, $v$ is Poisson's ratio. The pristine Young's modulus is chosen from experiments from Jackman et al. on the related material $\mathrm{Li}_{1.3} \mathrm{Al}_{0.3} \mathrm{Ti}_{1.7}\left(\mathrm{PO}_{4}\right)_{3}$ (LATP) [4]. Although this is a different composition than LAGP, it is expected that the Young's modulus values will be similar because these materials feature the same crystal structures and similar composition. The reacted interphase should be significantly less stiff, as the reaction process involves incorporation of a large quantity of lithium and $\sim 130 \%$ volume expansion [5], and pure Li has a lower Young's modulus. Since no experimental data is available for the material 
properties, we have chosen a value of $\sim 10 \%$ of $E_{\text {pristine, with subsequent sensitivity analysis showing that }}$ this choice does not significantly affect the simulation outcomes. The quantity $\Omega c_{R \text {,max }}$ gives rise to the volumetric chemical expansion, which is measured from cross-sectional SEM images such as that in Fig. 4a to be approximately $130 \%$. We thus use $\Omega c_{\mathrm{R}, \max }=1.3$ here. Finally, the maximum molar concentration $c_{\mathrm{R}, \max }$ is prescribed to be $0.0484 \times 10^{6} \mathrm{~mol} / \mathrm{m}^{3}$ based on estimates of Li concentration given the measured volume expansion.

As discussed above, the inelastic (plastic) behavior is incorporated to capture the inelastic flow (due to bond breaking and reforming) that occurs during the chemical reaction between Li and LAGP. To capture this process from a phenomenological perspective we chose the simplest flow rule characterized by

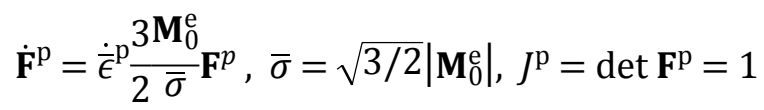

Here, the rate of change of the plastic distortion $\dot{\mathbf{F}}^{\mathrm{p}}$ is in the direction of the deviatoric Mandel stress ( $\mathbf{M}_{0}^{\mathrm{e}}$ ) and has a magnitude proportional to the equivalent plastic strain rate $\dot{\bar{\epsilon}}^{\mathrm{p}}$. Further, $\bar{\sigma}$ is the Mises equivalent stress, and $J^{\mathrm{p}}=\operatorname{det} \mathbf{F}^{\mathrm{p}}=1$ denotes that the plastic flow is incompressible. We then employ a no-flow condition when $\bar{\sigma} \leq Y$, where $Y$ is the yield stress. During plastic flow, the equivalent tensile stress is taken to be given by a rate-dependent flow strength of the form

$$
\bar{\sigma}=Y+Y_{*}\left(\dot{\bar{\epsilon}}^{p} / \dot{\epsilon}_{0}\right)^{1 / m}
$$

where $Y_{*}>0$ is a positive-valued stress-dimensioned constant, $\dot{\epsilon}_{0}$ is a reference strain rate, and $m$ is a measure of the strain-rate sensitivity of the material. We may summarize this by writing that the equivalent tensile plastic strain rate is given by

$$
\dot{\bar{\epsilon}}^{p}=\left\{\begin{array}{cc}
0 & \text { if } \bar{\sigma} \leq \mathrm{Y}_{0} \\
\epsilon_{0}\left(\frac{\bar{\sigma}-Y}{Y_{*}}\right)^{m} & \text { if } \bar{\sigma}>\mathrm{Y}_{0}
\end{array}\right.
$$

In this work, rate-dependence of the plastic flow is introduced for purely numerical reasons, since using elastic-perfectly-plastic behavior as an alternative leads to convergence difficulties. The only physical parameter in this model is the yield stress, which is taken to be given by $Y_{0}=500 \mathrm{MPa}$ purely from previous understanding of the mechanical behavior of other plastically-flowing materials during lithiation, such as $\mathrm{Si}$ [3].Sensitivity studies on this parameter will be presented subsequently. The rate-dependent parameters, which again are added purely for numerical stabilization and do not significantly affect the physics of the theory are given by 


$$
Y_{*}=50 \mathrm{MPa}, \dot{\epsilon_{0}}=1.0 \times 10^{-3}, m=3.00
$$

Finally, we wish to suppress plastic flow in the pristine LAGP phase since it was observed to undergo fracture. Since the two phases here are tracked through the normalized concentration $\bar{c} \in[0,1]$ with $\bar{c}=0$ being the pristine material and $\bar{c}=1$ being the fully reacted material, we chose to suppress plasticity by replacing the yield strength $Y$ with concentration-dependent flow of the form

$$
=Y_{\mathrm{f}}+\left(Y_{0}-Y_{\mathrm{f}}\right) \exp \left(\begin{array}{c}
\hat{Y}(\bar{c}) \\
\left.-\bar{c} / c_{*}\right)
\end{array}\right.
$$

where $Y_{f}$ is a very large stress quantity (taken as $Y_{f}=50 \mathrm{GPa}$ ) and $c_{*}$ is chosen to be 0.05 , a low value, such that plasticity is quickly activated as the reaction occurs. Mathematically, this choice for $\hat{Y}(\bar{c})$ suppresses plasticity in the unreacted material while maintaining a constant value of $Y_{0}$ within the reacted phase.

The diffusion of species is governed by mass balance, which may be expressed as $\dot{\bar{c}}=-J \operatorname{Div}(\mathbf{j})$, with $J=$ $\operatorname{det} \mathbf{F}$ and with the spatial flux of species $\mathbf{j}$ related through a mobility $m$ to the spatial gradient of the chemical potential $\mu$ through

$$
\begin{gathered}
\mathbf{j}=-m(\operatorname{grad} \mu), m=\frac{D_{0}}{R \theta} \bar{c}(1-\bar{c}) \\
\mu=R \theta \ln \left(\frac{\bar{c}}{1-\bar{c}}\right)-\frac{1}{3} \Omega\left(\operatorname{tr} \mathbf{M}^{\mathrm{e}}\right)
\end{gathered}
$$

Here, $D_{0}$ is the diffusivity, $R$ the gas constant, and $\theta$ the absolute temperature. We note that $\mu$, the chemical potential of the diffusing species, depends on the state of stress.

\section{Modeling of the evolution of the reacted interphase}

As noted in the main text, our focus is on predicting stress evolution due to the growth of the interphase and not on the actual physics of the reaction/deformation process. As such, we use the theory summarized above to develop the desired interphase region separated from the pristine LAGP by a sharp interface, as well as the corresponding stress state.

In order to obtain a relatively sharp reaction interface, we use a spatially-varying diffusivity and we define the location of the interface in advance. We then evolve the simulation to generate the chemically-induced elastic-plastic expansion and deformation and the associated induced stresses. Various works in the 
literature make use of spatially-varying diffusivity [3, 6] or concentration-dependent diffusivity [7-12] to reproduce a reaction interface without direct modeling of the reaction process.

We assume a spatially-varying diffusivity with the following function (known as logistic function and also related to the hyperbolic tangent)

$$
D(\boldsymbol{x})=D_{0}+\frac{D_{\mathrm{f}}-D_{0}}{1+\exp \left(-W\left(\boldsymbol{x}-\boldsymbol{x}_{\text {interface }}\right)\right)}
$$

Here, $D_{\mathrm{f}}$ is the diffusivity in the reacted region and is taken to be much larger than $D_{0}$, which is the diffusivity of the pristine material. The quantity $\boldsymbol{x}$ denotes the coordinate of interest and the quantity $\boldsymbol{x}_{\text {interface }}$ is hence the desired location of the interface. At the surface of the simulation domain at which contact with $\mathrm{Li}$ is simulated (i.e., where flux is prescribed - see Fig. S13 above), $\boldsymbol{x}$ is a coordinate perpendicular to the surface with a value of zero at the surface and positive in the downward direction (i.e. into the bulk). At the edge of the surface of the simulation domain where the flux boundary condition ends, $\boldsymbol{x}$ is prescribed as a radial coordinate originating at the surface. This gives rise to the rounded corner of the reacted phase, as seen in Fig. S15.

The values of $D_{f}$ and $D_{0}$ affect the theory only in their relation to each other and to the magnitude of the applied flux. We require that $D_{\mathrm{f}} \gg D_{0}$ and that the ramp rate of the applied flux be slower than the diffusion time $\tau=t^{2} / D_{\mathrm{f}}$ required to diffuse the length of the interface thickness $t$. Finally, the quantity $W$ in the definition of $D(\boldsymbol{x})$ above determines the width of the reaction front and of course the quantity $\boldsymbol{x}_{\text {interface }}$ sets the location of the interface and hence its thickness $t$. We emphasize that these diffusivity values should be thought of as related to the diffusion of atomic lithium into the LAGP to form the reacted interphase, and not to the conductions of ions through the LAGP as in standard battery operation.

The specific values used in simulation are given by

$$
D_{0}=10^{-14} \frac{\mathrm{m}^{2}}{\mathrm{sec}^{\prime}}, \quad D_{\mathrm{f}}=10^{4} D_{0}, B=100 .
$$

To highlight how we evolve the simulation to the final result, Fig. S15 shows contours of concentration (left) and profiles of concentration vs. vertical distance (right) for the simulation shown in the main text as the prescribed reacted interphase expands chemically to its final state. In this figure, the computational steps $\tau_{1}$ through $\tau_{3}$ are not to be considered physical, as they simply serve to evolve the simulation to the final step $\tau_{4}$ where the pre-defined reacted interphase is fully chemically expanded and has generated the associated stresses. To model the evolution of stress in time we perform multiple simulations with 
increasing interface thickness $t$ (which is modeled by changing the location $\boldsymbol{x}_{\text {interface}}$ ), these results are shown in Fig. 4 in the main text.
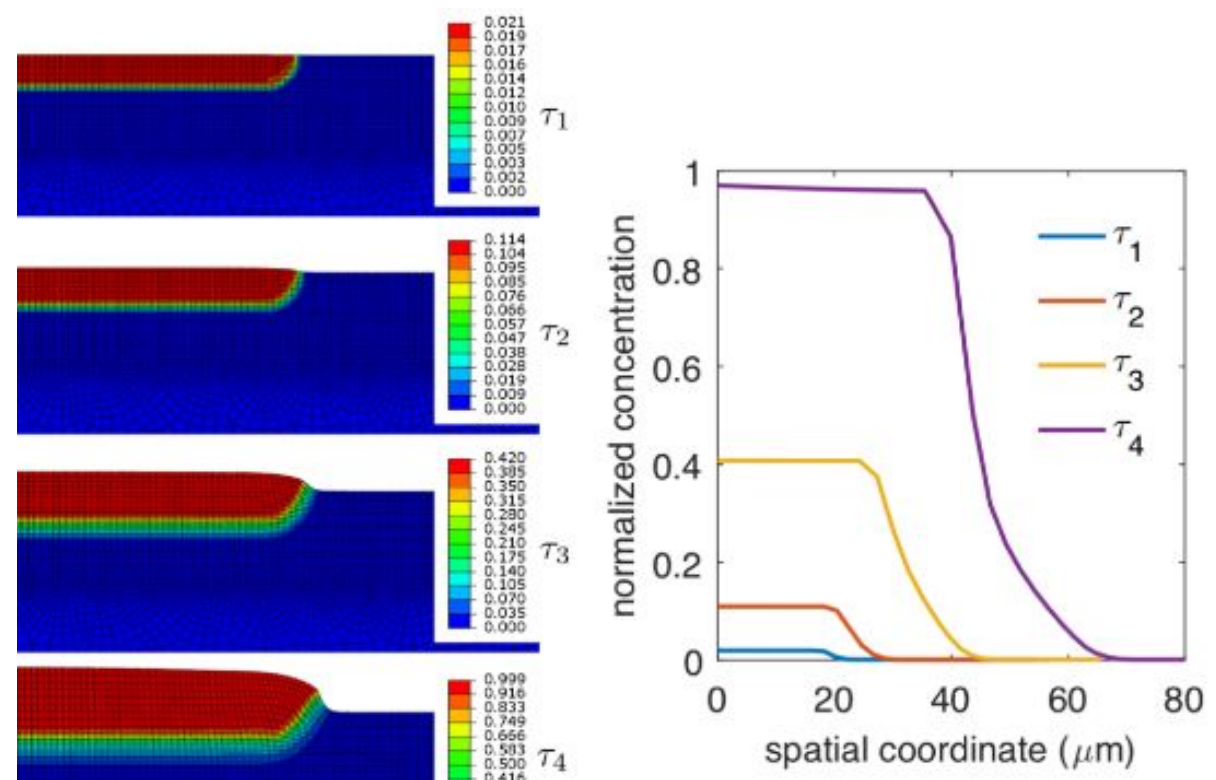

Figure S15: Evolution of concentration profile along the reacted interphase. The plot shows concentration vs. vertical distance into the LAGP pellet. Note that the color scales are different in each of the images.

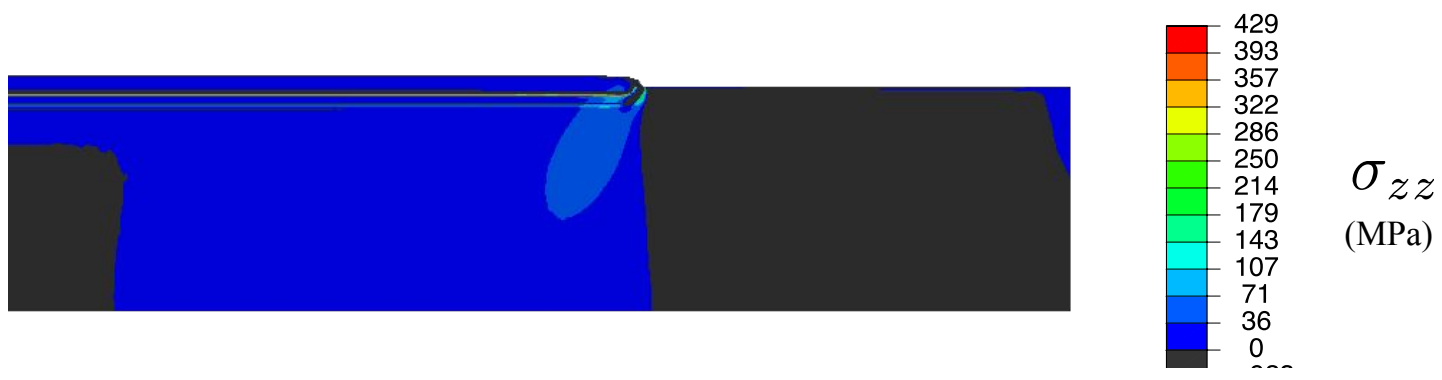

Figure S16: Axial stress $\left(\sigma_{\mathrm{zz}}\right)$ contours from the simulation; all regions showing negative (compressive) stress are shown in black. 


\section{Sensitivity Analysis}

As noted in the main text, the finite element results serve mainly to provide an understanding of the manner in which stresses develop within this material and how these stresses could lead to fracture. The magnitudes of these predicted stresses should be interpreted judiciously for two main reasons. First, fracture will occur at early times during the growth of the reacted interphase, as seen in the experiments. This would in turn alter the stress state in the material. Second, much remains to be understood about the mechanical properties and mechanical behavior of pristine and reacted LAGP.

It is useful to provide sensitivity analysis detailing the effects of varying some of the parameters in the aforementioned theory on stress distribution and magnitude in these simulations. Fig. S17 shows simulations that vary the Young's modulus of the unreacted (pristine) material, where the middle column shows the baseline case with $E_{\text {pristine }}=115 \mathrm{GPa}$ and the left and right columns show a decrease and increase of $20 \%$ from this baseline, respectively. Varying the Young's modulus by this amount does not have a significant effect on the magnitude or distribution of any of the stress components. Similar results are shown in Fig. S18 for variations in the Young's modulus of the reacted interphase ( $\left.E_{\text {reacted }}\right)$, and again no significant effect is observed. Both the magnitude and the distribution of stresses remain similar as this quantity is varied. Note that in all results presented, only positive stress values are shown and all negative values are represented as black contours. This, in accordance with Fig. 4, highlights the positive stresses which are most important in predicting the possible onset of fracture.

Figure S19 shows a comparison of different simulations in which the yield stress of the reacted interphase $Y_{0}$ was varied. Little is known of the inelastic mechanical behavior of LAGP during its chemical reaction with $\mathrm{Li}$, and as such understanding the sensitivity of the simulations to this parameter is important. As $Y_{0}$ is decreased, the overall magnitude of the stress decreases accordingly, while increasing $Y_{0}$ increases the magnitude of stresses in the material. This is expected, as plastic flow limits the maximum stresses that can be generated in the material during the coupled deformation-reaction process. Importantly, the specific value of $Y_{0}$ does not affect the stress distribution within the material, as the concentrations of each stress component are found in the same locations for all cases. These results show that the yield stress of the reacted interphase is an important parameter for determining stress magnitudes within the SSE material, and reacted materials with lower strength are more suitable for damage-resistant systems. 
An additional sensitivity analysis was performed to study how switching from a soft interphase to a hard one affects stress distribution. To do so, we ran another simulation with a yield stress of $Y_{0}=1.5 \mathrm{GPa}$ and Young's modulus of $E_{\text {reacted }}=50 \mathrm{GPa}$ and compare to the baseline case in Fig. S20. Again, the same overall distribution of stresses is observed and the only difference lies in the magnitudes, where increasing yield stress leads to higher stress magnitudes.

Figure S21 shows results in which the amount of expansion of the reacted interphase, as prescribed by the quantity $\Omega c_{\max }$, is varied. The baseline case of $\Omega c_{\max }=1.3$ corresponds to an expansion of approximately $130 \%$ during chemical reaction (as measured experimentally from chemically-reacted samples), and in this analysis we increase and decrease this value by $40 \%$. As shown in Fig. S21, varying $\Omega c_{\max }$ by this amount impacts the distribution of stresses slightly. Although the contours change as this parameter is varied, we observe the same overall behavior, with hot spots of stress concentrated at the edge of the reacted interphase. Fig. S22 shows the reaction coordinate for the three simulations with varying $\Omega c_{\max }$ and assists in rationalizing the changes in stress distribution. As we change the maximum chemical expansion, we also affect the shape of the interphase between the reacted and pristine fronts; in particular, at the corner where the stress hot spots are generated.

The sensitivity analysis shown here supports the results and conclusions presented in the main text. In particular, this analysis shows that the predicted stress distribution is present for a large range of material properties, and that although the physics is not entirely characterized at this time, we can expect stress distributions of the form shown in this work. 


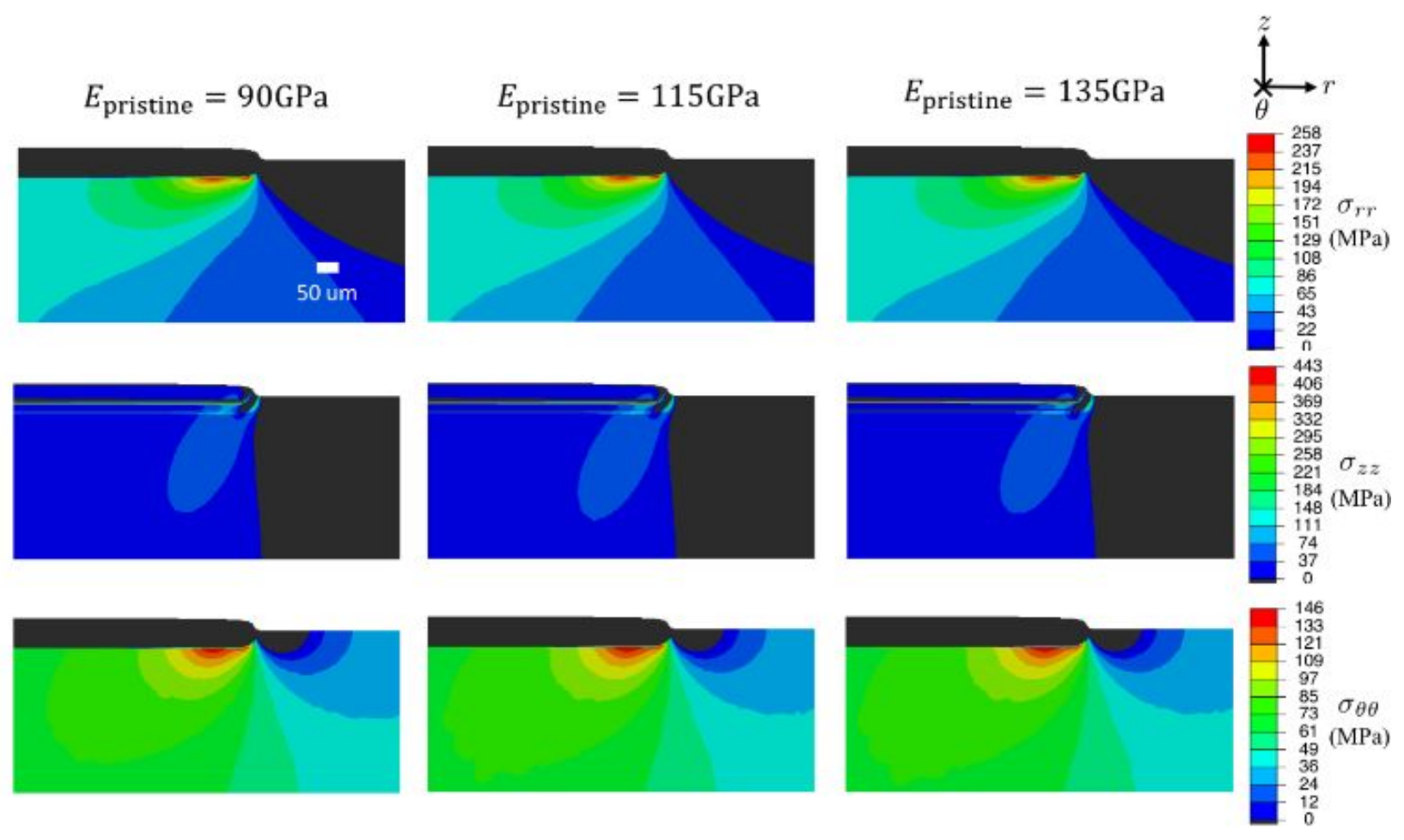

Figure S17: Effect of the Young's modulus of the pristine LAGP $\left(E_{\text {pristine }}\right)$ on stress contours. Note that the distribution and magnitude of the stress components remain similar from column to column. 


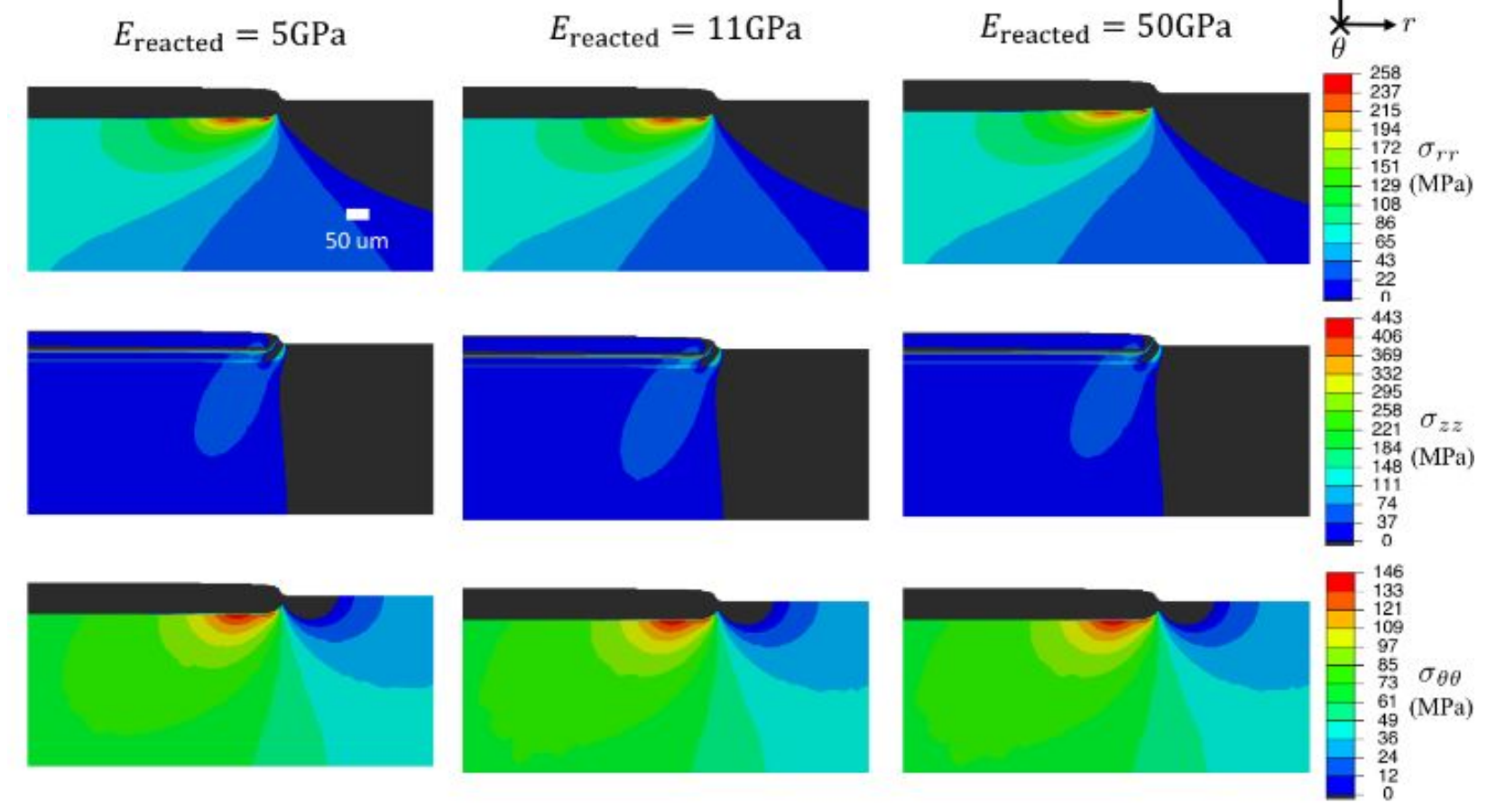

Figure S18: Effect of the Young's modulus of the reacted interphase $\left(E_{\text {reacted }}\right)$ on stress contours. Note that the distribution and magnitude of the stress components remain similar from column to column.
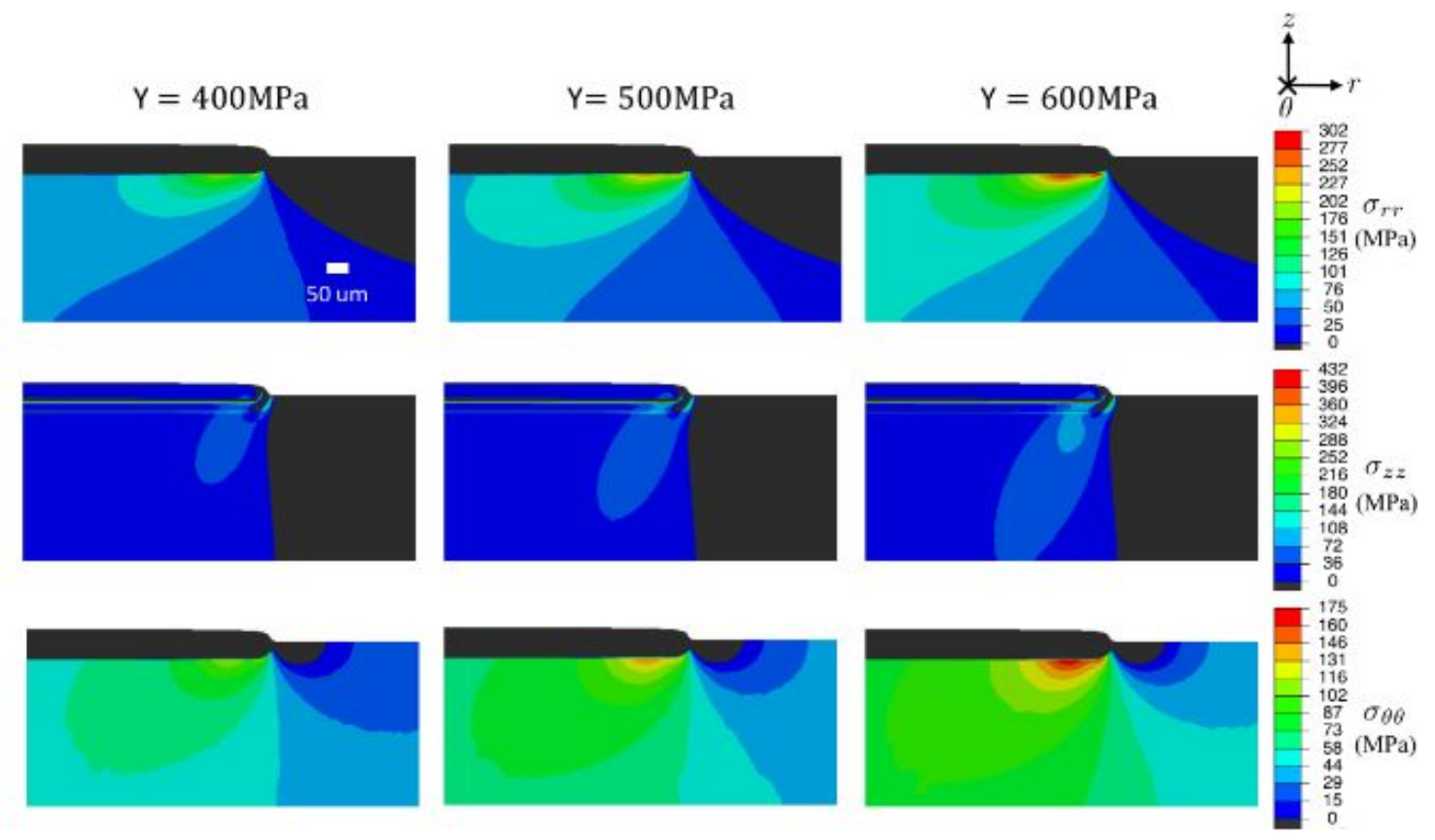

Figure S19: Effect of the yield stress $Y_{0}$ of the reacted interphase on stress contours. The distribution of stresses remains similar from column to column, while the magnitude of the stresses increases with increasing yield stress. 

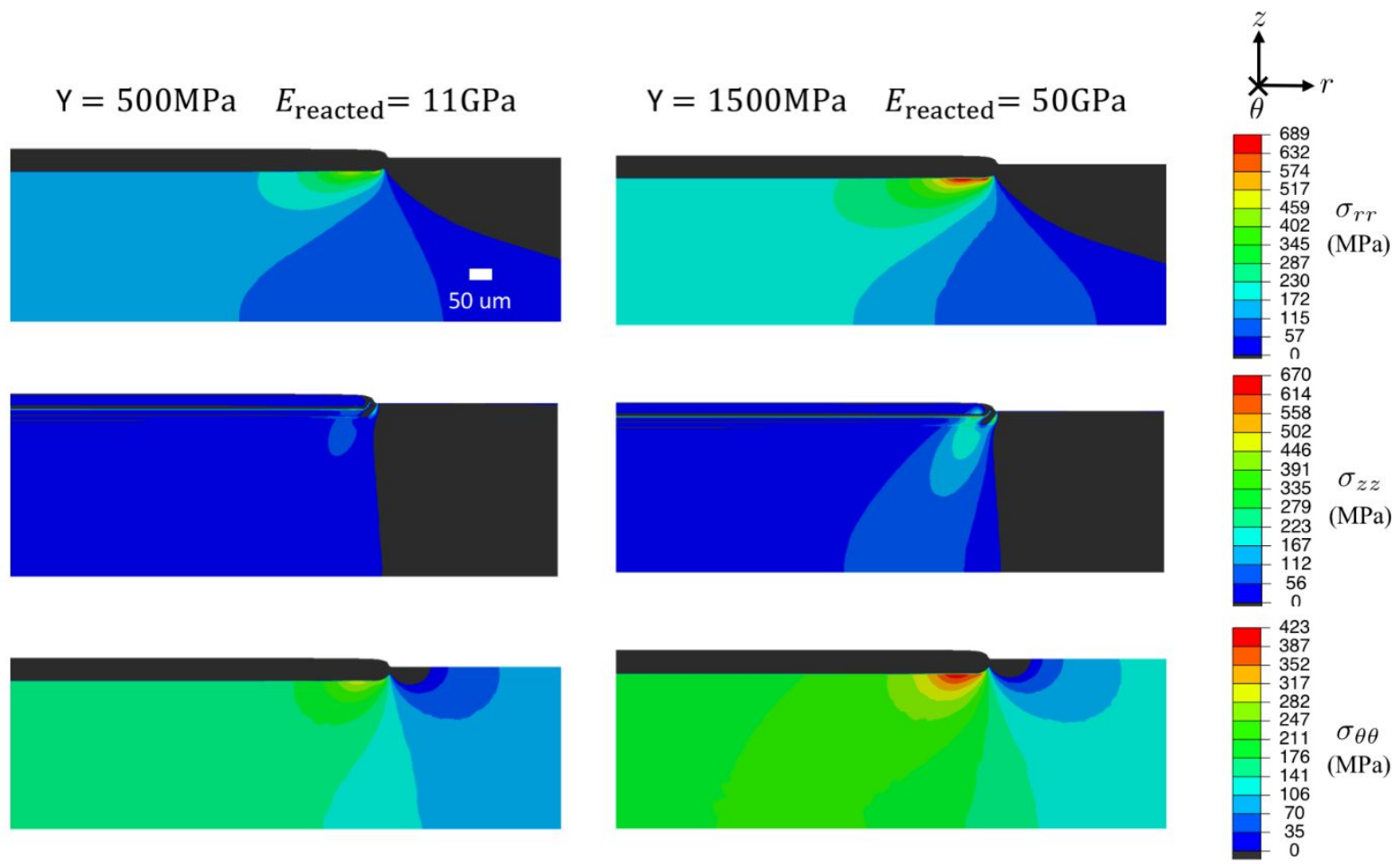

Figure S20: Effect of hard vs. soft reacted interphase on the stress contours. The distribution of stresses is the same for both cases, while the magnitude of the stresses increases for the harder interphase. 


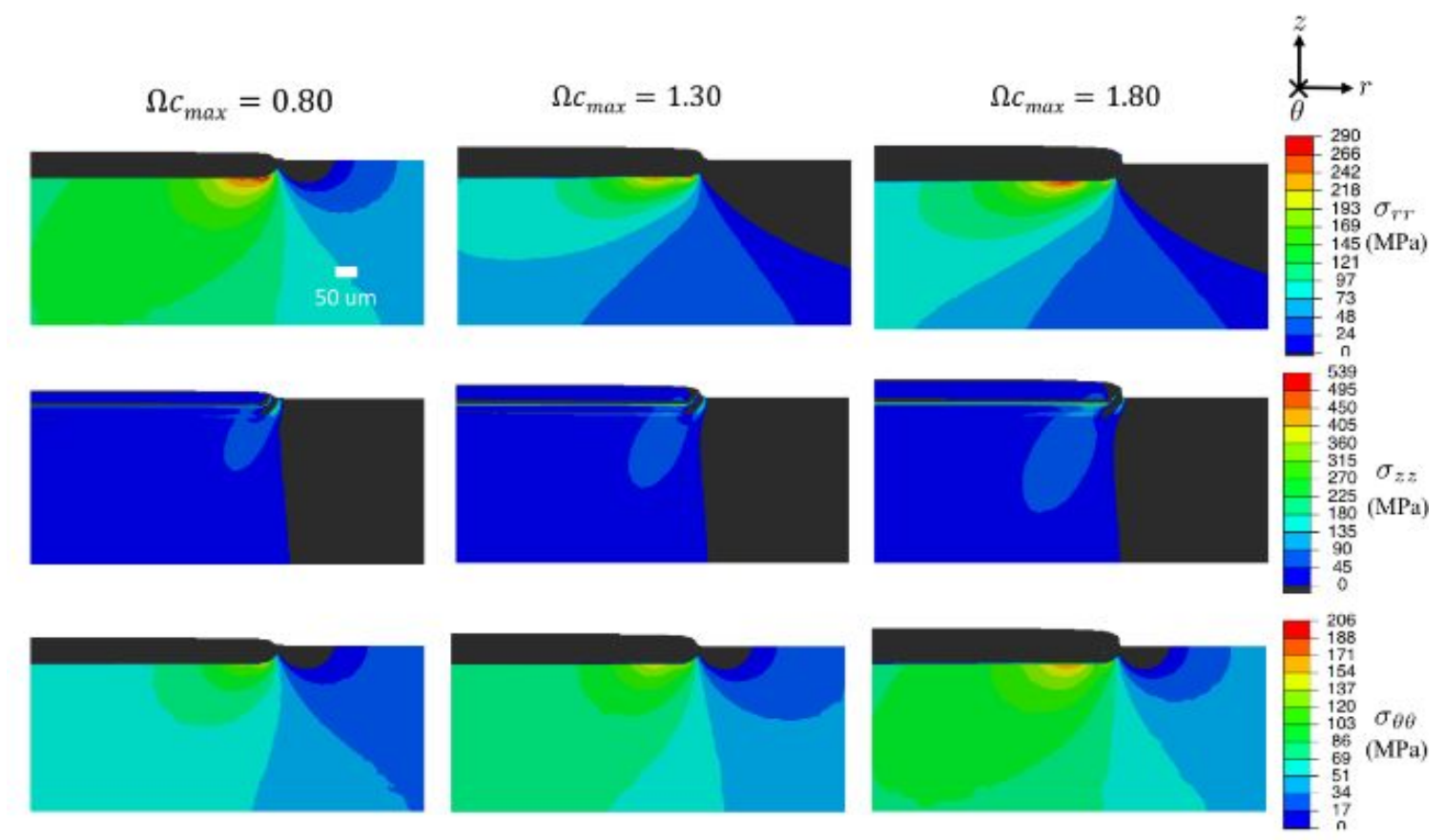

Figure S21: Effect of the magnitude of chemical expansion on stress contours. For all cases, similar stress distributions are observed, with concentrations of radial and circumferential stresses at the edge of the reacted interphase.

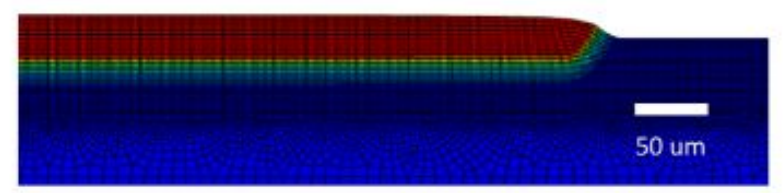

$$
\Omega c_{\max }=0.80
$$

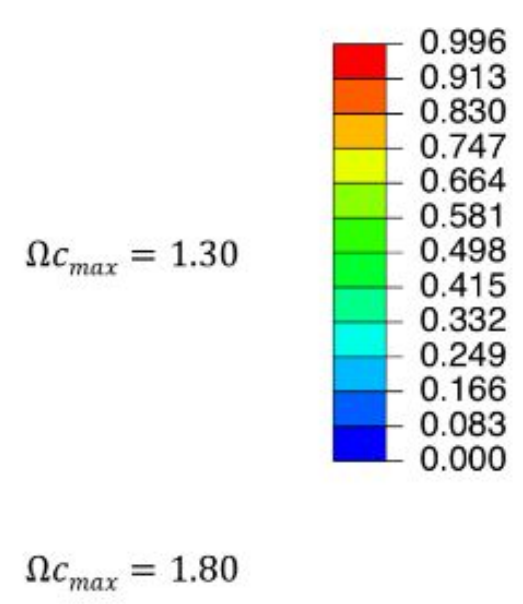

Figure S22: Volume change for the different values of chemical expansion shown in Fig. S21 depicted for full lithiation. 
(a)

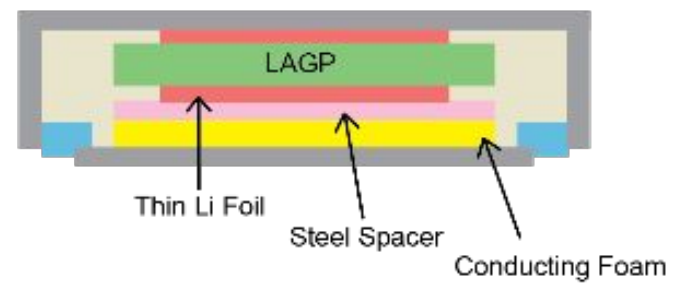

(b)

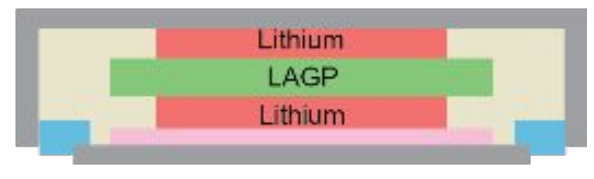

Figure S23: Coin cell architectures used in the experiments. (a) Cell construction used for the experiment in Fig. 2. (b) Cell construction used for the experiment in Fig. 3c-d.

\section{Supplemental References}

1. S. Kadioglu, F. Kharfi, A. C. Avelar, W. M. Ferreira, A. M. Menezes, S. Opoku, W. K. Antwi, S. R. Sarblah, H. A. Lylia \& M. G. Fonseca. in Imaging and radioanalytical techniques in interdisciplinary research-fundamentals and cutting edge applications; InTech, USA, 2013.

2. Di Leo, C. V., Rejovitzky, E. \& Anand, L. Diffusion-deformation theory for amorphous silicon anodes: The role of plastic deformation on electrochemical performance. Int. J. Solids Struct. 67-68, 283-296 (2015).

3. Sethuraman, V. A., Srinivasan, V., Bower, A. F. \& Guduru, P. R. In situ measurements of stress-potential coupling in lithiated silicon. J. Electrochem. Soc. 157, A1253-A1261 (2010).

4. Jackman, S. D. \& Cutler, R. A. Effect of microcracking on ionic conductivity in LATP. J. Power Sources 218, 65-72 (2012).

5. Lewis, J. A. et al. Interphase morphology between a solid-state electrolyte and lithium controls cell failure. ACS Energy Lett. 2, 591-599 (2019).

6. Huang, S., Fan, F., Li, J., Zhang, S. \& Zhu, T. Stress generation during lithiation of highcapacity electrode particles in lithium ion batteries. Acta Mater. 61, 4354-4364 (2013).

7. Zhang, S. Chemomechanical modeling of lithiation-induced failure in high-volume-change electrode materials for lithium ion batteries. npj Comput. Mater. 3, 7 (2017).

8. Yang, H. et al. A chemo-mechanical model of lithiation in silicon. J. Mech. Phys. Solids 70, 349-361 (2014).

9. Wang, C., Ma, Z., Wang, Y.\& Lu, C. Failure prediction of high-capacity electrode materials in lithium-ion batteries. J. Electrochem. Soc. 163, A1157-A1163 (2016).

10. Drozdov, A. \& Sommer-Larsen, P. Development of stresses in silicon nanolayer under lithiation. Modell. Simul. Mater. Sci. Eng. 22, 055009 (2014).

11. Wu, H., Xie, Z., Wang, Y., Lu, C. \& Ma, Z. Modeling diffusion-induced stress on twophase lithiation in lithium-ion batteries. Eur. J. Mech. A-Solid 71, 320-325 (2018).

12. Drozdov, A. Viscoplastic response of electrode particles in Li-ion batteries driven by insertion of lithium. Int. J. Solids. Struct. 51, 690-705 (2014). 